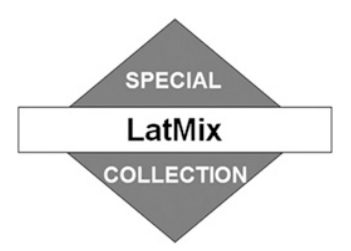

\title{
Note on the Rate of Restratification in the Baroclinic Spindown of Fronts
}

\author{
JÖRN CALLIES \\ California Institute of Technology, Pasadena, California \\ RAFFAELE FERRARI \\ Massachusetts Institute of Technology, Cambridge, Massachusetts
}

(Manuscript received 1 September 2017, in final form 21 March 2018)

\begin{abstract}
This paper revisits how the restratifying buoyancy flux $\overline{w^{\prime} b^{\prime}}$ generated by baroclinic mixed layer instabilities depends on environmental conditions. The frontal spindown is shown to produce buoyancy fluxes that increase significantly beyond the previously proposed and widely used scaling $\overline{w^{\prime} b^{\prime}} \sim f \Lambda^{2} H^{2}$ ( $f$ is the Coriolis parameter, $\Lambda$ is the geostrophic shear, and $H$ is the mixed layer depth), irrespective of whether the initial front is broad or narrow. This increase occurs after the initial phase of the nonlinear evolution, when the baroclinic eddies grow in size and develop velocities significantly in excess of the scaling assumption $V \sim \Lambda H$. Implications for parameterizing the restratification caused by baroclinic mixed layer instabilities in coarseresolution models are discussed.
\end{abstract}

\section{Introduction}

Submesoscale baroclinic instabilities are thought to be an important source of stratification in the surface ocean (e.g., Haine and Marshall 1998; Boccaletti et al. 2007). Mixed layer baroclinic instabilities at density fronts slide dense under light water, a process that tends to flatten isopycnal surfaces. A positive tendency in stratification is induced by a positive eddy buoyancy flux $\overline{w^{\prime} b^{\prime}}$, where $w^{\prime}$ and $b^{\prime}$ are the vertical velocity and buoyancy anomalies associated with the instability. The overline is an appropriately defined along-front average. Since baroclinic mixed layer instabilities occur on small horizontal scales of order $0.1-10 \mathrm{~km}$, they are not typically resolved by presently available global ocean models. The eddies' effects on stratification and transport must be parameterized.

Fox-Kemper et al. (2008, hereinafter FFH) proposed to represent the slumping of isopycnals by baroclinic mixed layer instabilities using an eddy streamfunction, similar to how the slumping of isopycnals by mesoscale eddies in the thermocline is often parameterized (Gent and McWilliams 1990). To set the rate of mixed layer restratification, FFH proposed a scaling for the buoyancy flux:

Corresponding author: Jörn Callies, jcallies@caltech.edu
FFH: $\overline{w^{\prime} b^{\prime}} \sim f \Lambda^{2} H^{2}$,

where $f$ is the Coriolis parameter, $\Lambda$ is the geostrophic shear associated with the lateral buoyancy gradient of the mixed layer front, and $H$ is the mixed layer depth. The geostrophic shear is defined as a vertical and alongfront average of the instantaneous geostrophic shear.

FFH considered the restratification by mixed layer baroclinic instabilities as an initial-value problem: they tested the scaling (1) in a suite of spindown experiments of mixed layer fronts. In these experiments, it was assumed that a mixed layer had been created in a lateral buoyancy front, which in turn had been generated by mesoscale straining. It was further assumed that the atmospheric forcing that had created the mixed layer had subsided and that the mesoscale straining that had created the front had ceased. The analysis focused on the subsequent restratification of the mixed layer in response to baroclinic instability. Such a transient restratification process has been observed at the end of the winter season, when mixed layer instabilities lead to the final shoaling of deep mixed layers (e.g., Mahadevan et al. 2012). The test cases of FFH and Bachman and Fox-Kemper (2013) suggest that the scaling (1) is optimal in the sense that there is no or only weak dependence on 
(a)

$$
\hat{t}=37
$$

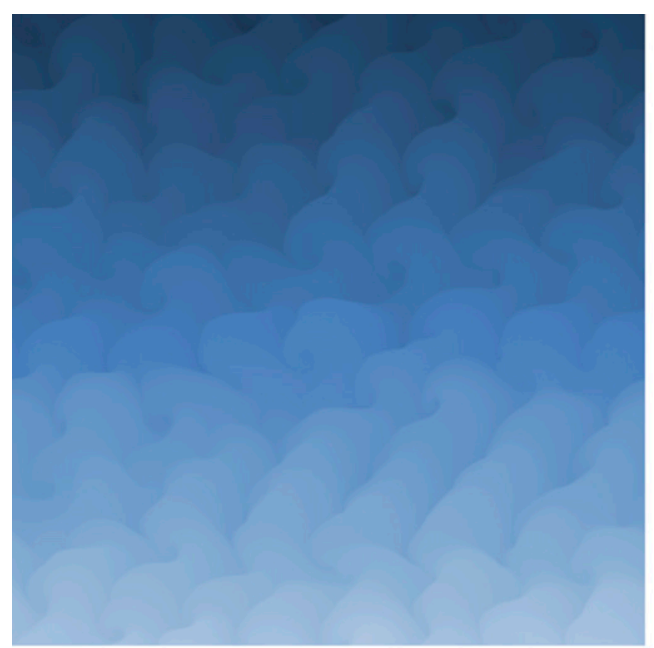

(c)

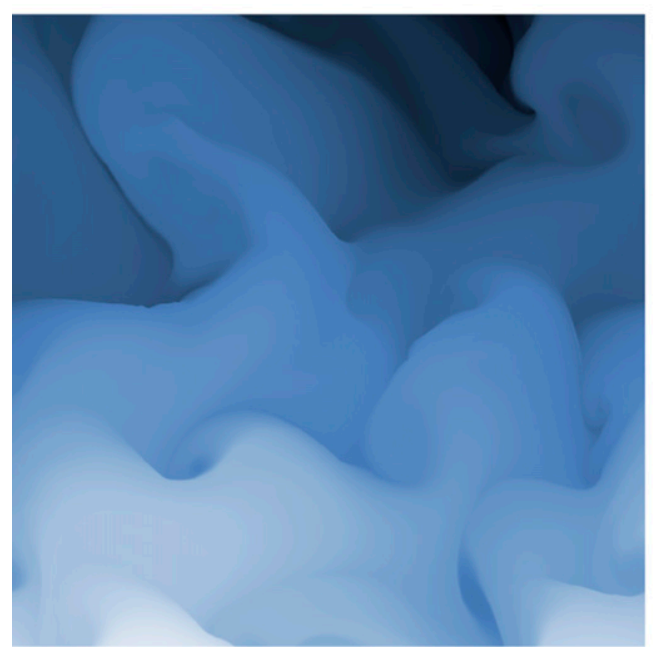

(b)

$$
\hat{t}=57
$$

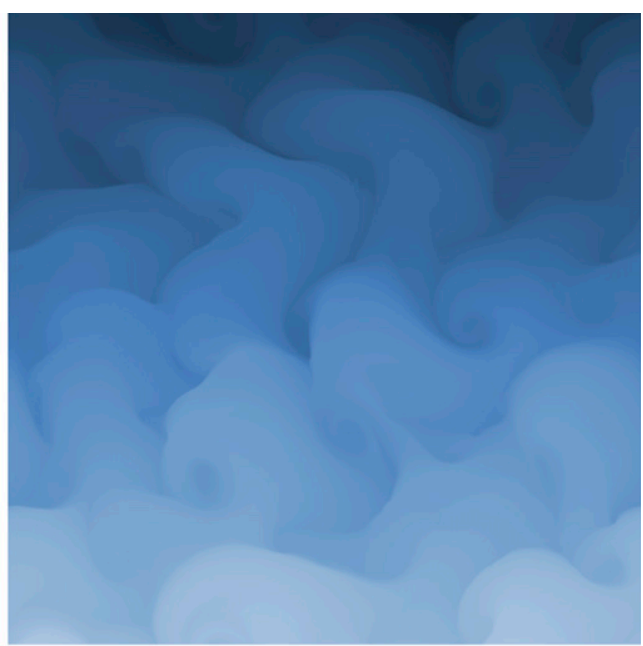

(d) $\hat{t}=97$

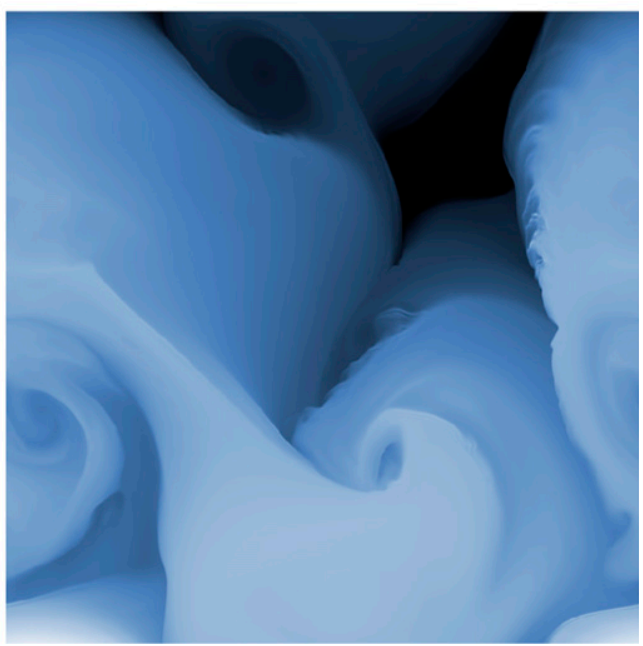

FIG. 1. Snapshots of surface buoyancy $-\hat{y}+\hat{b}$ showing the evolution of the broad front in the widest domain $(\hat{L}=40)$. The color scale ranges between $\pm \hat{L}$ from white through blue to black.

other parameters of the initial conditions, such as the Richardson number.

Considering two cases of frontal spindown, we show in this paper that the scaling (1) captures the buoyancy flux produced by baroclinic instabilities only in the initial phase of the nonlinear evolution. Subsequently, the buoyancy flux increasingly exceeds the scaling as the eddies grow in size and eddy velocities exceed the scaling assumption $V \sim \Lambda H$ (cf. Bachman and Fox-Kemper 2013). This transient increase beyond the scaling occurs both in a front that is much broader than the size of the developing baroclinic eddies (Fig. 1) and in a front that is initially narrow and subsequently broadened by the evolving eddies (Fig. 4), suggesting the increase is independent of the width of the front. Implications for the parameterization of baroclinic mixed layer eddies are discussed in the conclusions.

\section{Broad front}

In a frontal zone that is much broader than the eddies generated by baroclinic instability, there is no horizontal eddy flux divergence, so the mean lateral buoyancy gradient remains unchanged. The parameters in the scaling (1) therefore remain fixed, and the vertical buoyancy flux is predicted to be constant.

We test this prediction in a set of simulations in which the meridional buoyancy gradient $-f \Lambda$ (in thermal wind balance with a zonal current with vertical shear $\Lambda$ ) is imposed and remains fixed. Such a frontal-zone setup 
has been used extensively to study quasigeostrophic baroclinic turbulence (e.g., Bretherton and Karweit 1975; Salmon 1980; Haidvogel and Held 1980; Larichev and Held 1995) and submesoscale processes (e.g., Taylor and Ferrari 2010; Callies and Ferrari 2018). It assumes an infinite scale separation between the buoyancy gradient and the baroclinic eddies. The other extreme-the case in which the buoyancy gradient is concentrated into a narrow front-is examined in the next section.

We work in the nondimensional variables

$$
\begin{aligned}
& \hat{t}=f t, \quad \hat{x}=\frac{f x}{\Lambda H}, \quad \hat{y}=\frac{f y}{\Lambda H}, \quad \hat{z}=\frac{z}{H}, \\
& \hat{u}=\frac{u}{\Lambda H}, \quad \hat{v}=\frac{v}{\Lambda H}, \quad \hat{w}=\frac{w}{f H}, \quad \hat{b}=\frac{b}{\Lambda^{2} H}, \quad \hat{p}=\frac{p}{\Lambda^{2} H^{2}},
\end{aligned}
$$

where $u, v$, and $w$ are the zonal, meridional, and vertical velocity components, respectively; $b$ is buoyancy; $p$ is pressure divided by a reference density; and $H$ is the domain depth. The nondimensional background meridional buoyancy gradient is then -1 , and the nondimensional zonal background shear is 1 . The Boussinesq equations for perturbations from this background flow are

$$
\begin{aligned}
\hat{u}_{\hat{t}}+\hat{z} \hat{u}_{\hat{x}}+\hat{w}+\hat{\mathbf{u}} \cdot \hat{\nabla} \hat{u}-\hat{v} & =-\hat{p}_{\hat{x}}+\mathscr{D} \hat{u}, \\
\hat{v}_{t}+\hat{z} \hat{v}_{\hat{x}}+\hat{\mathbf{u}} \cdot \hat{\nabla} \hat{v}+\hat{u} & =-\hat{p}_{\hat{y}}+\mathscr{D} \hat{\boldsymbol{v}}, \\
\delta^{2}\left(\hat{w}_{\hat{t}}+\hat{z} \hat{w}_{\hat{x}}+\hat{\mathbf{u}} \cdot \hat{\nabla} \hat{w}\right) & =\hat{b}-\hat{p}_{\hat{z}}+\delta^{2} \mathscr{D} \hat{w}, \\
\hat{b}_{\hat{t}}+\hat{z} \hat{b}_{\hat{x}}-\hat{v}+\hat{\mathbf{u}} \cdot \hat{\nabla} \hat{b} & =\mathscr{D} \hat{b}, \quad \text { and } \\
\hat{\nabla} \cdot \hat{\mathbf{u}} & =0,
\end{aligned}
$$

where $\mathscr{D} A=\mathrm{Ek}_{h} \delta^{2}\left(A_{\hat{x} \hat{x}}+A_{\hat{y} \hat{y}}\right)+\mathrm{Ek}_{v} A_{\hat{z} \hat{z}}$ defines the friction/diffusion operator (different horizontal and vertical Ekman numbers are allowed; the Prandtl number is set to one), and $\delta=f / \Lambda$ is Stone's (1971) nonhydrostatic parameter. All perturbations are assumed to be doubly periodic in the horizontal in a square domain of zonal and meridional extent $\hat{L}=f L / \Lambda H$, where $L$ is the dimensional domain width. The vertical boundary conditions are no normal flow $(\hat{w}=0)$, no stress on the perturbations $\left(\hat{u}_{\hat{z}}=\hat{v}_{\hat{z}}=0\right)$, and no buoyancy flux $\left(\hat{b}_{\hat{z}}=0\right)$ at $\hat{z}=0$ and $\hat{z}=-1$. For simplicity, we replace the strongly stratified thermocline by a solid bottom boundary, a good approximation as long as the thermocline stratification is much stronger than the mixed layer one (cf. Garner et al. 1992). There is an implied stress $\pm E k_{v}$ at the top and bottom that maintains the mean flow against dissipation. For the small $\mathrm{Ek}_{v}$ of the simulations discussed below, these implied stresses have a negligible effect on the energetics of the flow that develops. For a full discussion of the energetics, see Callies and Ferrari (2018).
The scaling (1) involves only prescribed parameters, and it takes the nondimensional form

$$
\text { FFH: } \quad \overline{\hat{w}^{\prime} \hat{b}^{\prime}} \sim \text { const. }
$$

The along-front average in (1) is equivalent to a domain average, because the broad-front system is statistically homogeneous in the horizontal. The overbar in (9) thus denotes a volume average over the entire domain. Using the MITgcm (Marshall et al. 1997), we perform a suite of experiments with a fixed set of these parameters-we only vary the domain size $\hat{L}$. The scaling (9) predicts no dependence on the domain size, as long as the unstable modes fit into the domain.

We choose $\delta=1$, such that the baroclinic instability is in the hydrostatic regime (Stone 1971), and we initialize the flow with $\hat{b}_{\hat{z}}=\mathrm{Ri}=1$ and zero perturbation velocities. This initial state is stable to symmetric instability, and the most unstable mode is baroclinic (Stone 1966). A numerical linear stability analysis yields a wavelength of $\hat{\lambda}=5.5$ for the most unstable mode (Stone 1970, 1971). The instability is kicked off by small random initial perturbations in $\hat{b}$ of magnitude 0.25 . The horizontal and vertical Ekman numbers are $\mathrm{Ek}_{h}=6.25 \times 10^{-3}$ and $\mathrm{Ek}_{v}=1.25 \times 10^{-3}$. The domain sizes and time steps are $\hat{L}=5$ with $\Delta \hat{t}=10^{-2}, \hat{L}=10$ with $\Delta \hat{t}=5 \times 10^{-3}, \hat{L}=20$ with $\Delta \hat{t}=2.5 \times 10^{-3}$, and $\hat{L}=40$ with $\Delta \hat{t}=1.25 \times 10^{-3}$. For increasing domain size, the time step must be decreased, because larger velocities occur in larger domains, as described below. The grid spacing is $\Delta \hat{x}=\Delta \hat{y}=\Delta \hat{z}=0.05$ in all cases.

As expected, the instability develops first at the scale of the most unstable mode in all simulations. Larger eddies are subsequently energized until they reach the size of the domain (Fig. 1). This increase in eddy size is quantified by diagnosing the dominant wavelength $\hat{\lambda}_{0}$ defined by

$$
\frac{2 \pi}{\hat{\lambda}_{0}}=\frac{\iint \hat{k_{h}} \hat{S}(\hat{k}, \hat{l}) d \hat{k} d \hat{l}}{\iint \hat{S}(\hat{k}, \hat{l}) d \hat{k} d \hat{l}}
$$

where $\hat{k}_{h}=\left(\hat{k}^{2}+\hat{l}^{2}\right)^{1 / 2}, \hat{k}$ and $\hat{l}$ are the zonal and meridional wavenumbers, $\hat{S}$ is the vertically averaged horizontal buoyancy variance spectrum, and the integration is over the entire wavenumber space. The dominant eddy size increases and then saturates at a value that depends on the domain size (Fig. 2a). The increase in eddy size is expected for a number of reasons: larger-scale modes reach finite amplitude later, as they have smaller growth rates; smaller-scale modes become stable as restratification occurs and the cutoff scale for the instability increases; and larger eddies can be 

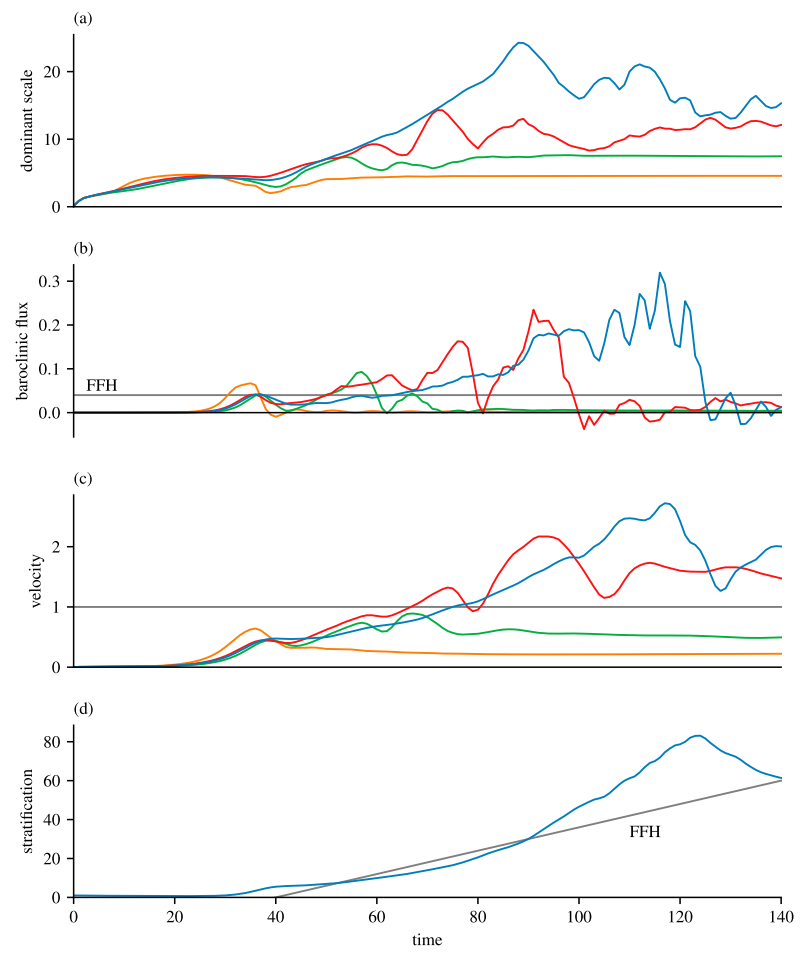

$\hat{L}=40$
$-\hat{L}=20 \quad \hat{L}=10$
$-\hat{L}=5$

FIG. 2. Broad-front regime time series of (a) the dominant wavelength $\hat{\lambda}_{0}$, (b) the buoyancy flux $\hat{\hat{w}^{\prime} \hat{b}^{\prime}}$, (c) the root-meansquare meridional velocity, and (d) the average stratification $\hat{b}_{\hat{z}}$ for simulations with different domain sizes. Also shown in (b) and (d) are the predictions of FFH given by (11) and (13) with an arbitrary intercept (gray lines). Also shown in (c) is the maximum mean zonal velocity (gray line).

energized through a nonlinear transfer of energy across scales.

In all simulations, the baroclinic instability eventually subsides. This is due to the increase in stratification, which shuts off baroclinic growth by pushing the short-wave cutoff beyond the domain scale, such that no unstable mode fits into the domain anymore. The domain size imposes a limit on the size of the baroclinic eddies. In the following discussion, we thus focus on the growth phase, in which the domain size is not a limiting factor and active baroclinic instability converts potential to kinetic energy.

Irrespective of the processes that energize larger eddies over the course of this growth phase, the scaling (9) predicts the vertical buoyancy flux to remain constant once the eddies have reached finite amplitude. Specifically, the FFH parameterization predicts

$$
\text { FFH: } \quad \overline{\hat{w}^{\prime} \hat{b}^{\prime}}=0.06 \overline{\mu(\hat{z})}=0.04,
$$

given the vertical profile

$$
\mu(\hat{z})=\left[1-4\left(\hat{z}+\frac{1}{2}\right)^{2}\right]\left[1+\frac{5}{3}\left(\hat{z}+\frac{1}{2}\right)^{2}\right] .
$$

Again, the overbar denotes a volume average over the domain.

After an initial phase of approximate agreement, the simulations deviate from this prediction (Fig. 2b). There is a significant increase beyond the value predicted by (11) as the eddies grow in size - if their growth is not limited by the domain size. The increase of the flux is intermittent and occurs in bursts associated with the eddy growth at discrete low wavenumbers, but a clear pattern stands out: growth of the eddy size and eddy kinetic energy (Fig. 2c) is followed by an increase in buoyancy flux. The maximum flux is achieved when eddies have reached the domain scale. As a consequence, there is an increase in maximum buoyancy flux as the domain size is increased. The increase beyond the prediction (11) is largest for the largest domain. When the size of the eddies reaches the domain scale, the instability shuts off and $\overline{\hat{w}^{\prime} \hat{b}^{\prime}}$ drops to zero.

The increase in eddy size over the course of the growth phase goes hand in hand with an increase in eddy velocities (Fig. 2c). The root-mean-square meridional eddy velocity increases beyond the maximum velocity of the mean flow, which violates the assumption $\hat{V} \sim 1$ made in the derivation of (1) and (9).

The simultaneous increase in buoyancy flux and eddy velocities beyond their respective scaling is consistent with the result of Bachman and Fox-Kemper (2013). They showed that the buoyancy flux can be better captured if the eddy velocity scale is known as an input to the flux prediction. The simulations presented here suggest that this is a significant effect if there is time for the eddies to grow significantly beyond their initial size.

A buoyancy flux that increases in time leads to accelerating restratification, which is clearly visible in the largest domain (Fig. 2d). This acceleration is not captured by the FFH prediction, which implies a constant increase in domain-average stratification:

$$
\mathrm{FFH}: \quad \overline{\hat{b}_{\hat{z} \hat{t}}}=-0.06 \overline{\mu_{\hat{z} \hat{z}}(\hat{z})}=0.6 .
$$

The disparity in the restratification rate is largest when eddies have grown largest. For even larger domain sizes, the stratification rate would likely surpass the prediction (13) even more dramatically. The domain size dependence is artificial, but it reveals a dependence of the eddy flux on the evolving eddy size and velocity scale.

\section{Narrow front}

The simulations presented by FFH to corroborate the scaling (1) passed from the broad-front to the 
narrow-front regime: the eddies grew to a size comparable to the initial width of the frontal zone, began to broaden the front, and started to decrease the mean lateral buoyancy gradient. If the scaling (1) is applied to the evolving frontal zone, it predicts the vertical buoyancy flux to rapidly decrease once the narrow-front regime is reached and the mean lateral buoyancy gradient has started to decrease. We show in this section, however, that the buoyancy flux in the frontal zone does not decrease nearly as strongly as required by the scaling. The narrow-front regime thus also exhibits an increase of the buoyancy flux beyond the prediction (1) as the baroclinic eddies grow in size. Eddy velocities again increasingly exceed the scaling assumption $V_{f} \sim \Lambda_{f} H$ as the front broadens, where $V_{f}$ and $\Lambda_{f}$ are the evolving eddy velocity scales and mean zonal shear in the frontal zone.

We perform a simulation of the spindown of a front that is initialized to be a few times narrower than the initial instability scale. We use the same doubly periodic setup as above, but we choose an initial buoyancy perturbation that cancels out the background buoyancy gradient in the bulk of the domain to confine it to a narrow front of width $\hat{L}_{f}$ (Fig. 3):

$$
\hat{b}=\operatorname{Ri}\left(\hat{z}+\frac{1}{2}\right)+\hat{y}-\frac{\hat{L}}{2} \tanh \frac{2 \hat{y}}{\hat{L}_{f}} .
$$

The buoyancy jump across the front is $\hat{L}$, such that the buoyancy anomalies at $\hat{y}= \pm \hat{L} / 2$ very nearly match $\left(\hat{L}_{\mathrm{f}} \ll \hat{L}\right)$ and periodicity is ensured. The initial zonal flow perturbation is in thermal wind balance,

$$
\hat{u}_{\hat{z}}=-1+\frac{\hat{L}}{\hat{L}_{f}} \operatorname{sech}^{2} \frac{2 \hat{y}}{\hat{L}_{f}},
$$

and we set $\hat{u}=0$ at $\hat{z}=-1$. Both the lateral buoyancy gradient and the zonal flow are very weak outside the frontal zone. This setup can be thought of as an infinite succession of fronts spaced a distance $\hat{L}$ apart, with the constraint that all fronts evolve identically. The setup behaves like one with walls at $\hat{y}= \pm \hat{L} / 2$, as used by FFH, as long as there is no flow there.

The most unstable baroclinic mode of a narrow front is expected to occur around the same wavenumber $\hat{\lambda}$ as predicted by Stone (1966) (cf. Juckes 1998), which for small Richardson numbers and in our nondimensionalization scales with the frontal shear, $\hat{\lambda} \approx 4 \hat{\Lambda}_{f}=\hat{L} / \hat{L}_{f}$. We thus set $\hat{L}_{f}=\hat{\Lambda}_{f}$ for an initial frontal width a few times narrower than the instability scale. We further set the frontal nonhydrostatic parameter $\delta_{f}=\delta / \hat{\Lambda}_{f}=1$, as in the broad-front case. To get a large separation between the initial instability scale and the domain size, we (a)

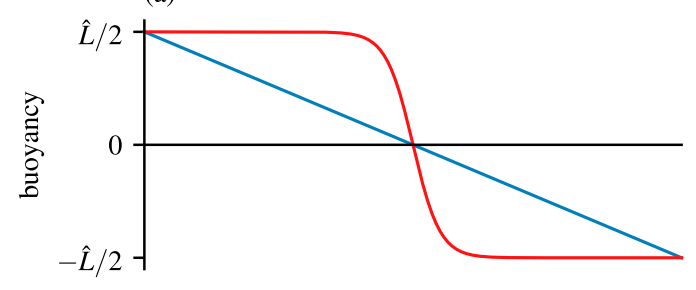

(b)

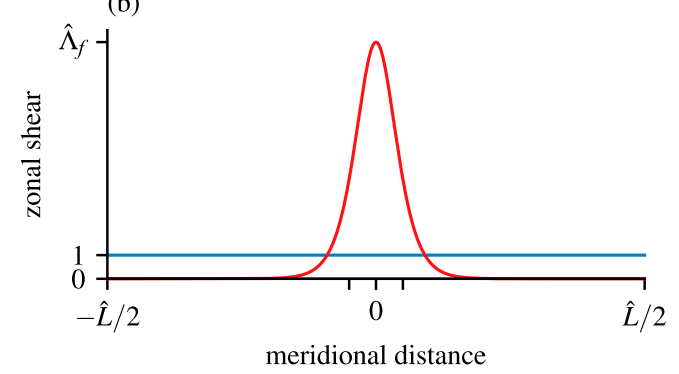

FIG. 3. The initial meridional profiles of (a) buoyancy $-\hat{y}+\hat{b}$ at $\hat{z}=-1 / 2$ and (b) zonal shear $1+\hat{u}_{\hat{z}}$ for the broad-front (blue) and narrow-front (red) setups. Note that the anomaly $\hat{b}$ vanishes in the broad-front case and is periodic in the narrowfront case. The unlabeled tick marks on the horizontal axis are at $\pm \hat{L}_{f} / 2$.

choose $\hat{L}=6400, \hat{L}_{f}=80$, and $\delta=80$. Since the mixed layer is weakly stratified, we set $\mathrm{Ri}=1$. The horizontal and vertical Ekman numbers are $\mathrm{Ek}_{h}=6.25 \times 10^{-4}$ and $\mathrm{Ek}_{v}=6.25 \times 10^{-5}$. The time step is $\Delta \hat{t}=10^{-2}$ and the grid spacing is $\Delta \hat{x}=\Delta \hat{y}=4$ and $\Delta \hat{z}=0.05$.

In the frontal zone, where the shear is enhanced and the local Richardson number is below one, symmetric instabilities develop rapidly (Fig. 4a). This occurs before the baroclinic mode has reached appreciable amplitude, and the frontal zone is rapidly adjusted to a state of zero potential vorticity (e.g., Emanuel 1994; Haine and Marshall 1998; Taylor and Ferrari 2009). A pulse in the buoyancy flux around $\hat{t}=15$ is associated with this adjustment (Fig. 5a). Once the stratification has been adjusted in the frontal zone, the symmetric mode becomes subdominant and the baroclinic mode takes over (cf. Haine and Marshall 1998). ${ }^{1}$

\footnotetext{
${ }^{1}$ Symmetric instabilities can be avoided by increasing the initial stratification to the point that the local Richardson number in the front is one, which can be achieved by setting $\mathrm{Ri}=6400$. That choice does not qualitatively change any of the results presented in this section, but it gives a regime that is not relevant for the ocean mixed layer. The initial stratification is so strong that buoyancy fluxes associated with baroclinic eddies do not appreciably affect the stratification.
} 
(a)

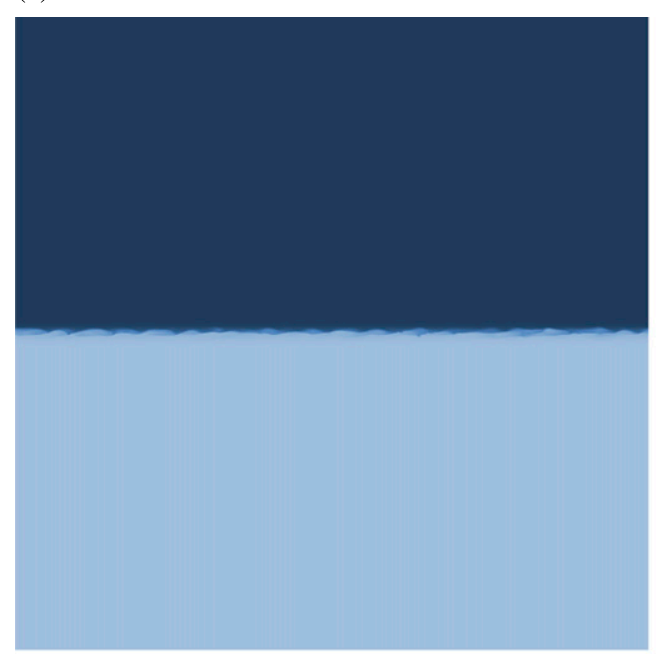

(c)

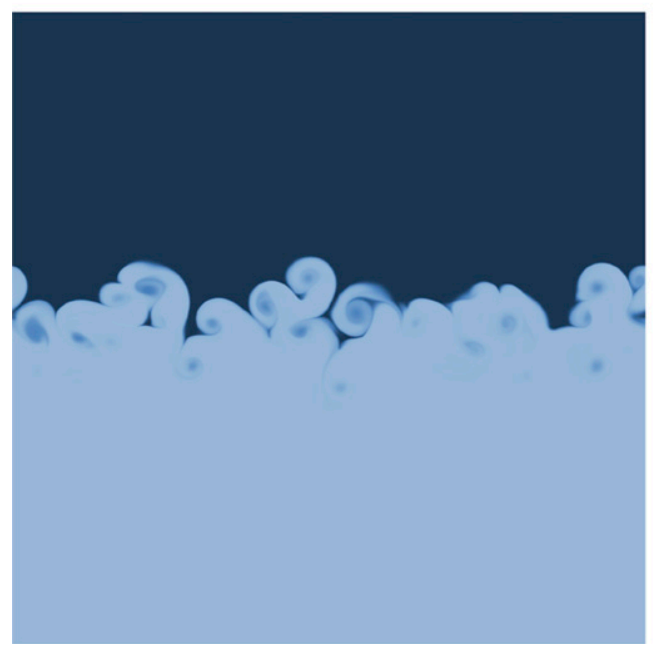

(b)

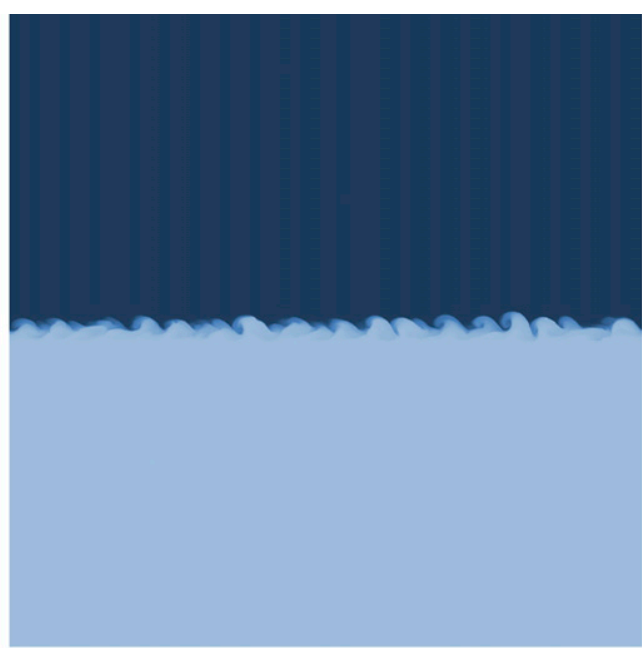

(d)

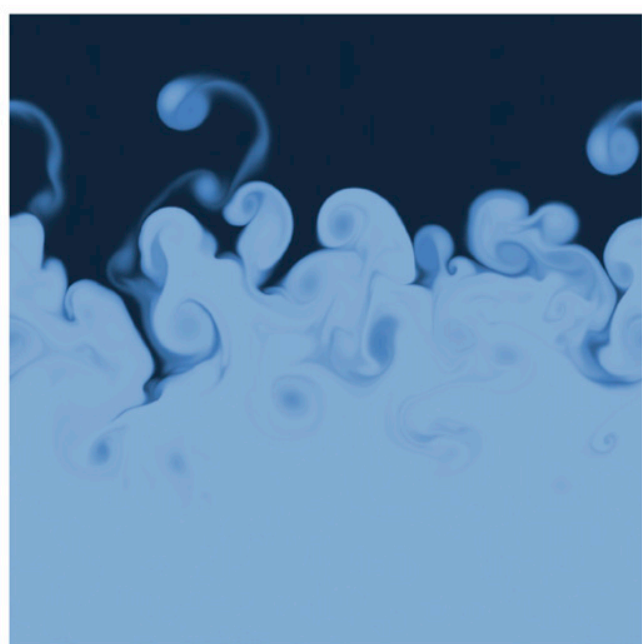

FIG. 4. Snapshots of surface buoyancy $-\hat{y}+\hat{b}$ showing the evolution of the narrow front. The color scale ranges between $\pm \hat{L}$ from white through blue to black.

As expected, the baroclinic instability occurs initially at a wavelength of about 360 , a few times larger than the initial frontal width $\hat{L}_{f}=80$ (Fig. 4b). Fully nonlinear baroclinic eddies subsequently develop and increase in scale as they flatten the front (Figs. 4c,d). The eddies increase the frontal scale $\hat{L}_{f}$, which subsequently is of the same order as the eddy size (cf. Haine and Marshall 1998; Manucharyan and Timmermans 2013). We stop the simulation before the eddies fill the domain, at which point the periodicity of the setup would affect the further development of the front. The analysis is restricted to the period in which the eddies widen the front into undisturbed fluid.

We diagnose the domain-average buoyancy flux $\overline{\hat{w}^{\prime} \hat{b}^{\prime}}$. After the initial pulse due to symmetric instabilities, this flux increases as the baroclinic instability grows, and it subsequently remains roughly constant (Fig. 5a). As explained in the following, this again implies that the buoyancy flux in the frontal zone increases beyond what is predicted by the scaling (1) as the eddies grow in size and velocities exceed $\hat{V}_{f} \sim \hat{\Lambda}_{f}$, where $\hat{V}_{f}$ is the nondimensional eddy velocity scale in the frontal zone.

Applying the scaling (1) to the frontal zone, that is, using the frontal-zone geostrophic shear and averaging over the frontal zone only, yields the prediction

$$
\text { FFH: } \quad \hat{\hat{w}}^{\prime} \hat{b}^{\prime} \sim \hat{\Lambda}_{f}^{2},
$$

where the overbar with a superscript " $f$ " denotes a volume average restricted to the frontal zone, and $\hat{\Lambda}_{f}$ 

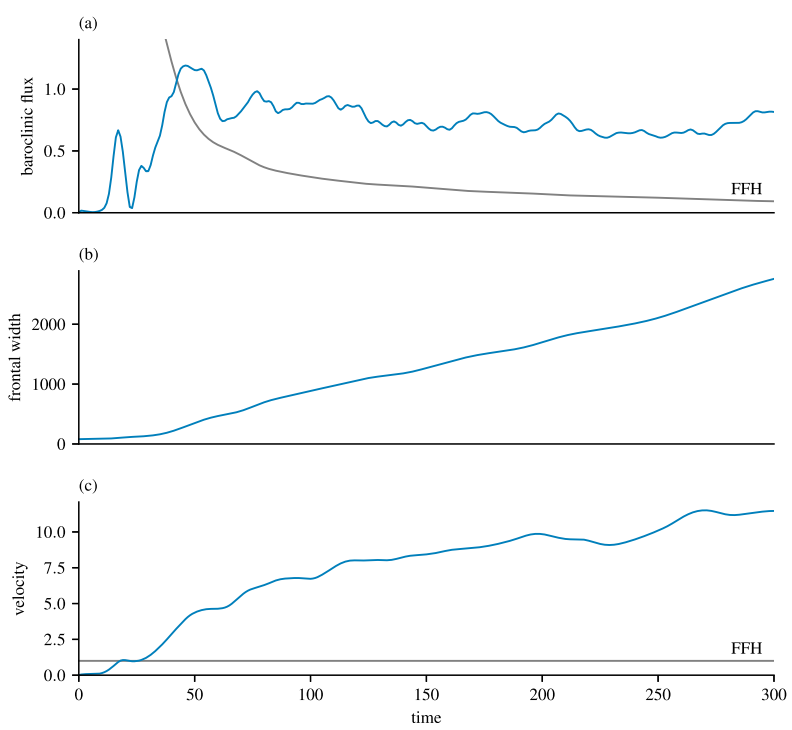

FIG. 5. Narrow-front regime time series of (a) the domain-averaged buoyancy flux $\hat{w}^{\prime} \hat{b}^{\prime}$, (b) the diagnosed frontal width $\hat{L}_{f}$, and (c) the domain-average root-mean-square meridional velocity.

denotes the evolving meridional buoyancy gradient averaged vertically and over the frontal zone. ${ }^{2}$ Since the buoyancy difference $\hat{L}$ across the front remains fixed, the buoyancy gradient in the frontal zone of evolving width $\hat{L}_{f}$ scales like

$$
\hat{\Lambda}_{f} \sim \frac{\hat{L}}{\hat{L}_{f}} .
$$

We can relate the frontal-zone average of the buoyancy flux to its domain average, because the flux is largely confined to the frontal zone:

$$
\overline{\hat{w}^{\prime} \hat{b}^{\prime}} \sim \frac{\hat{L}_{f}}{\hat{\hat{L}}^{\prime} \hat{\underline{b}}^{\prime}} .
$$

The scaling (16) for the buoyancy flux in the frontal zone then yields the prediction

$$
\text { FFH: } \quad \overline{\hat{w}^{\prime} \hat{b}^{\prime}} \sim \frac{\hat{L}}{\hat{L}_{f}} .
$$

The domain-average buoyancy flux is thus predicted to decrease like $\hat{L}_{f}^{-1}$ as the front broadens.

We diagnose the frontal width $\hat{L}_{f}$ in our simulation by fitting a function of the form $-(\hat{L} / 2) \tanh \left(2 \hat{y} / \hat{L}_{f}\right)$ to the

\footnotetext{
${ }^{2}$ We follow Fox-Kemper and Ferrari (2008) in using the timeevolving buoyancy gradient, averaged vertically and over the frontal zone-not the initial value of $\Lambda_{f}$.
}

vertically and zonally averaged buoyancy field $-\hat{y}+\hat{b}$. As expected from the snapshots (Fig. 4), the diagnosed frontal width increases by over an order of magnitude from its initial value $\hat{L}_{f}=80$ to a value comparable with the domain size $\hat{L}=6400$ (Fig. 5b). The prediction from (19) thus amounts to a sharply decreasing domainaverage buoyancy flux, which is inconsistent with the diagnosed flux (Fig. 5a).

To get a prediction for the magnitude of the domainaverage buoyancy flux, we restore the proportionality constant in (19):

$$
\text { FFH: } \quad \overline{\hat{w}^{\prime} \hat{b}^{\prime}}=0.04 \frac{\hat{L}}{\hat{L}_{f}},
$$

where we integrated the vertical profile in (12) as we did in (11). With the diagnosed $\hat{L}_{f}$, the predicted flux roughly matches the diagnosed flux around $\hat{t}=50$, when the baroclinic eddies first become nonlinear (Fig. 5a). Subsequently, the diagnosed flux increasingly exceeds the prediction (20) as the eddies grow larger and the front wider (Fig. 5a). The increase of the buoyancy flux beyond the prediction is qualitatively consistent with that found in the broad-front regime.

We similarly diagnose a transient increase in the eddy velocities in the frontal zone beyond the scaling assumption

$$
\text { FFH: } \quad \hat{V}_{f} \sim \hat{\Lambda}_{f} \sim \frac{\hat{L}}{\hat{L}_{f}} .
$$

Given this assumption and the fact that nonzero velocities are largely confined to the frontal zone, the domain-average eddy velocity scale $\hat{V}$ is predicted to be constant,

$$
\text { FFH: } \quad \hat{V} \sim \frac{\hat{L}_{f}}{\hat{L}} \hat{V}_{f} \sim 1 .
$$

In violation of the assumption (21), the diagnosed domainaverage root-mean-square meridional velocity instead significantly increases over the course of the simulation (Fig. 5c). Like in the broad-front regime, eddy velocities thus show a transient increase beyond the scaling assumption $\hat{V}_{f} \sim \hat{\Lambda}_{f}$.

It should be noted that if the scaling (19) is to be used in a parameterization, the frontal width $\hat{L}_{f}$ must be specified. The implementation of Fox-Kemper et al. (2011, hereinafter FK11) is based on (19), but the mixed layer deformation radius is substituted for the frontal width. This changes the scaling behavior compared to (19) with the actual frontal width $\hat{L}_{f}$, the consequences of which are discussed in the appendix. 


\section{Discussion}

The numerical experiments presented here suggest that the buoyancy flux $\overline{w^{\prime} b^{\prime}}$ increases beyond (1) as the baroclinic eddies evolve after they have first reached finite amplitude. This increase appears to be due to the increase in eddy velocities beyond the scaling assumption $V \sim \Lambda H$. The spindown of broad and narrow fronts exhibits the same behavior with respect to the scaling.

The tests performed by FFH and Fox-Kemper and Ferrari (2008) did not reveal the increase of the restratification rate beyond scaling (1). Two reasons appear to explain this result: first, the simulations spent limited time in the broad-front regime, as explained below, so the increase in $\overline{w^{\prime} b^{\prime}}$ was modest, and second, the diagnostics used in these papers did not reveal the decrease of the vertically averaged buoyancy gradient in the narrow-front regime, so the constancy of $\overline{w^{\prime} b^{\prime}}$ in this regime was possibly misinterpreted as consistent with the scaling (cf. Fig. 5).

The simulations of FFH and Fox-Kemper and Ferrari (2008) were initialized (in their reference setup) with a front that had a width 4 times larger than the instability scale. There was thus limited scope for the eddies to grow in size and for $\overline{w^{\prime} b^{\prime}}$ to increase before the system transitioned to the narrow-front regime. While modest, inconsistencies with the scaling do appear in the broadfront regime. In the comparison of Fox-Kemper and Ferrari (2008) between the evolution of a parameterized front with a full three-dimensional front, the restratifying streamfunction of the full simulation was about twice that of the parameterized version toward the end of the broad-front regime, signifying an increasing $\overline{w^{\prime} b^{\prime}}$ in the full simulation (Fig. 3 of Fox-Kemper and Ferrari 2008). The increasing streamfunction in the full simulation led to accelerating restratification not captured by the parameterization, but only over a limited time interval (Fig. 5 of Fox-Kemper and Ferrari 2008). These inconsistencies would have become much more apparent had the front been broader initially (cf. Fig. 2d).

As the simulations of FFH and Fox-Kemper and Ferrari (2008) entered into the narrow-front regime, the average $\overline{w^{\prime} b^{\prime}}$ stopped increasing (cf. Fig. 5a). As discussed above, this constancy of the flux was inconsistent with the scaling (1), because the bulk buoyancy gradient decreased as the frontal zone broadened. FFH and FoxKemper and Ferrari (2008), however, restricted their diagnosis of the buoyancy gradient to locations in the horizontal and vertical that had a large gradient, thus neglecting regions of weak gradient above and below the front, which would have contributed to a bulk, vertically averaged gradient. This appears to have led to the diagnosis of a buoyancy gradient that was roughly constant in time (equal to the initial gradient) and, when inserted into scaling (1), gave a prediction that was consistent with the approximately constant average $\overline{w^{\prime} b^{\prime}}$. This was the result of ignoring two effects in the scaling: the progressive decrease in the bulk buoyancy gradient and the concomitant increase in the velocity scale beyond $\Lambda_{f} H$.

While the scaling (1) does not capture the transient increase of the buoyancy flux, it does approximately match the flux in the initial phase of the nonlinear evolution. The results of FFH and Bachman and FoxKemper (2013) suggest that for this initial phase, there is no or only weak dependence of the flux on other parameters of the initial conditions, that is, on $\mathrm{Ri}$ and $\delta$. Scaling (1) should thus be considered accurate for the initial phase of the evolution, and it only fails thereafter.

\section{Conclusions}

The transient increase beyond scaling (1) in the spindown of both broad and narrow fronts raises the question of how and whether initial-value problems can inform the parameterization of mixed layer restratification. Is it appropriate to think of the restratification by mixed layer baroclinic instabilities as a set of initial-value problems initialized once storms have passed over and have mixed the upper ocean? Does atmospherically forced mixed layer turbulence reset the state of the upper ocean when the next storm passes? If that is the case, (1) can provide an accurate scaling for the buoyancy flux in the initial phase of the nonlinear evolution, which probably is long enough to cover the period between mixing events occurring every few days.

In Callies and Ferrari (2018), however, we showed that submesoscale eddies are remarkably resilient to the presence of atmospherically forced small-scale turbulence in the mixed layer. This resilience suggests that submesoscale eddies can survive the passage of a storm, such that the release of available potential energy in deep winter mixed layers can continually energize the submesoscale range. In such a scenario, mixed layer eddies equilibrate with mesoscale eddies through nonlinear energy exchange across scales and energy fluxes into the thermocline (Sasaki et al. 2014; Callies et al. 2016). The results of this paper suggest that it is unlikely that the buoyancy flux produced by baroclinic mixed layer eddies in such an equilibrium is captured by scaling (1), because it only applies to the initial phase of the nonlinear spindown of a mixed layer front. Whether the long-term behavior of the spindown problem explored in this paper is more representative of such an equilibrium is 
equally unclear, but it adds to our overall understanding of baroclinic restratification.

To properly address these questions, one has to understand the interaction between mesoscale eddies and baroclinic mixed layer instabilities, with a representation of small-scale mixed layer turbulence that does not unduly damp out baroclinic mixed layer eddies. ${ }^{3}$ The cascade dynamics discussed in Callies et al. (2016) may offer a path forward, suggesting a scenario in which the conversion from mean to eddy available potential energy in the mixed layer, which is achieved by resolvable mesoscale eddies, can be related to the unresolved vertical buoyancy flux due to baroclinic mixed layer eddies, because the eddy available potential energy cascades down to scales around the mixed layer deformation radius, where it is converted into kinetic energy by $\overline{w^{\prime} b^{\prime}}$ (cf. Larichev and Held 1995). The dynamics considered in Callies et al. (2016) were quasigeostrophic and thus neglected any impact of mixed layer instabilities on the stratification, so the mixed layer was artificially kept fixed. The evolution of the mixed layer in the presence of mesoscale and submesoscale eddies as well as atmospheric forcing requires further study.

The transient increase of the buoyancy flux in spindown experiments suggests that an equilibrated submesoscale eddy field may achieve much larger restratification rates than expected from scaling (1) and the small proportionality constant inferred from spindown experiments $(\mathrm{FFH})$. The realistically forced numerical simulations of Capet et al. (2008), which had a grid spacing fine enough to allow the development of baroclinic mixed layer instabilities, did produce buoyancy fluxes much larger than those predicted. It remains open, however, whether the resolution of these simulations was high enough to faithfully capture baroclinic mixed layer instabilities and whether the parameterized representation of atmospherically forced smallscale turbulence interacted with submesoscale eddies in a realistic way.

It is clear that an advective restratification akin to the Gent and McWilliams (1990) parameterization of mesoscale eddies is needed in coarse-resolution models in order to capture the effect of mixed layer

\footnotetext{
${ }^{3}$ Bachman and Taylor (2016) considered the equilibration of baroclinic eddies by vertical diffusion. In such an equilibrium, the vertical buoyancy flux exceeds (11) by about an order of magnitude, which is not inconsistent with the transient increase found in our test cases. Vertical diffusion, however, unlikely equilibrates mixed layer eddies in the real ocean (cf. Callies and Ferrari 2018), so our discussion here focuses instead on energy exchange across scales and into the thermocline.
}

eddies. Despite the questions emerging from the test cases presented here, the parameterization of FFH has improved the representation of upper-ocean properties in such models (FK11; Gent et al. 2011). Answering some of the remaining questions may improve our estimate for the restratification rate. It can thus be hoped that understanding and capturing the buoyancy flux in a broader set of circumstances will help further decrease biases in global ocean models.

Acknowledgments. Jean-Michel Campin is thanked for assistance in implementing the MITgcm modifications necessary to prescribe a background flow. Discussions with Baylor Fox-Kemper are gratefully acknowledged. Funding came from NSF Grant OCE1233832 .

\section{APPENDIX}

\section{FK11 Applied to the Narrow-Front Case}

In their implementation of the scaling (1) as a parameterization in coarse-resolution ocean models, FK11 had to relate the buoyancy gradient on the unresolved frontal scale to the buoyancy gradient on the coarse grid scale. They argued that for a collection of fronts that produces a buoyancy spectrum falling off steeply beyond a defined frontal scale $L_{f}$, the application of (1) yields the gridbox average

$$
\text { FFH: } \quad \overline{w^{\prime} b^{\prime}} \sim \frac{L}{L_{f}} f \Lambda^{2} H^{2},
$$

where $L$ is the size of the coarse grid box, which we identify with our domain size, and $\Lambda$ is the geostrophic shear on the scale of the coarse grid. This expression is equivalent to the narrow-front scaling (19), which we derived by applying (1) to the frontal zone and relating the frontal-zone average to the domain average.

To turn the expression (A1) into a parameterization, FK11 had to specify the frontal width $L_{f}$. They noted that observations suggest that buoyancy spectra start falling off steeply around the mixed layer deformation radius $L_{d}=\bar{b}_{z}^{1 / 2} H / f$, suggesting a frontal width $L_{f}=L_{d}$. In nondimensional variables, this substitution yields the prediction

$$
\text { FK11: } \quad \overline{\hat{w}^{\prime} \hat{b}^{\prime}}=0.04 \frac{\hat{L}}{\hat{L}_{d}},
$$

where the nondimensional deformation radius is $\hat{L}_{d}=$ $f L_{d} / \Lambda H$ and we integrated over the vertical profile. 

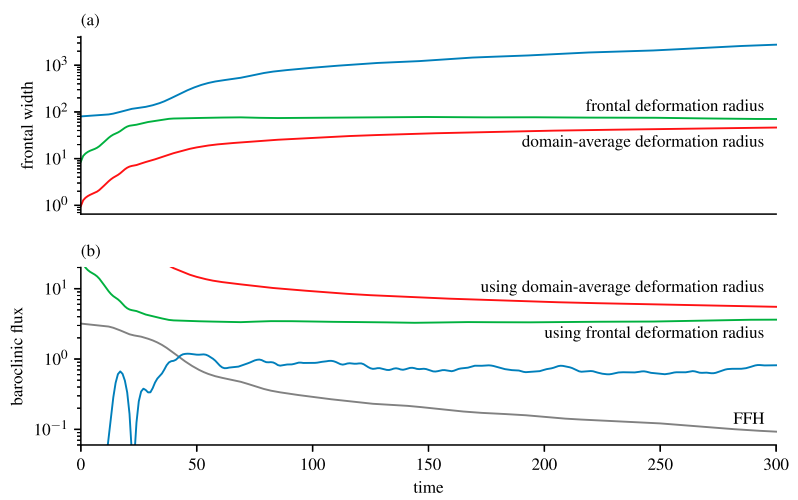

FIG. 6. Evaluating the FK11 parameterization in the narrowfront regime: (a) domain-average deformation radius $\hat{L}_{d}$ (red) and frontal deformation radius $\left(\hat{L} / \hat{L}_{f}\right)^{1 / 2} \hat{L}_{d}$ (green) vs the diagnosed frontal width $\hat{L}_{f}$ (blue), and (b) the domain-average buoyancy flux $\hat{\hat{w}^{\prime} \hat{b}^{\prime}}$ predicted by (A2) using the domain-average deformation radius (red) and the frontal deformation radius (green) vs the flux diagnosed from the model (blue). Also shown in (b) is the prediction (20) with the diagnosed frontal width $\hat{L}_{f}$ (gray), the same as in Fig. 5a.

Since the deformation radius does not necessarily match the actual frontal width, this reinterpretation of (1) yields a different scaling behavior.

In our narrow-front simulation, the domain-average deformation radius $\hat{L}_{d}$ does not match the width of the frontal zone (Fig. 6a). At all times, the deformation radius is at least an order of magnitude smaller than the diagnosed frontal width $\hat{L}_{f}$. Nevertheless, using it in (A2) drastically overpredicts the actual buoyancy flux (Fig. 6b).

This drastic overprediction is somewhat disconcerting, because it suggests that the FK11 parameterization might give restratification rates that are much too strong in situations resembling our test case. The parameter regime of our test case does not seem irrelevant for the real ocean's mixed layer, but it is quite an idealized case. The fact that the frontal width does not match the deformation radius in our simulation, while it does appear to match it in observations, means this result should be taken with a grain of salt. More realistic simulations are needed, in which the fronts are generated internally and mesoscale strain is present (see section 5).

The domain-average deformation radius depends on the stratification outside the front, which is not necessarily expected to affect the buoyancy fluxes within the frontal zone. A more sensible substitute for the frontal width in (A2) might thus be the deformation radius within the frontal zone. We diagnose the frontal deformation from the simulation as $\left(\hat{L} / \hat{L}_{f}\right)^{1 / 2} \hat{L}_{d}$, assuming the domain-average stratification is dominated by the frontal zone. This frontal deformation radius, however, is not a good predictor for the frontal width either
(Fig. 6a). Once the baroclinic instability has reached finite amplitude, the frontal deformation radius becomes roughly constant at a value around $\hat{L}^{1 / 2}=80$. This is expected, because the horizontal buoyancy difference across the front $\hat{L}$ is turned into a vertical buoyancy difference in the frontal zone.

The constancy of the frontal deformation radius thus does not capture the actual increase in the width of the frontal zone (Fig. 6a). Using the frontal instead of the domain-average deformation radius in (A2), however, does correctly predict a buoyancy flux that is roughly constant in time once the baroclinic instability has reached finite amplitude (Fig. 6b). The magnitude of the predicted flux is too large by a factor of about four. Whether this offset is systematic cannot be evaluated by a single simulation and should be checked by varying the parameters of the problem (initial $\hat{L}_{f}, \mathrm{Ri}$, and $\delta$ ).

If (A2), with $\hat{L}_{d}$ replaced by the frontal deformation radius, does turn out to be a good predictor for the domain-average buoyancy flux of a narrow front, it still remains unclear how that prediction could be used in a parameterization. One would have to relate the frontal deformation radius to grid-scale variables to yield a closed expression.

\section{REFERENCES}

Bachman, S., and B. Fox-Kemper, 2013: Eddy parameterization challenge suite I: Eady spindown. Ocean Modell., 64, 12-28, https://doi.org/10.1016/j.ocemod.2012.12.003.

_ _ and J. R. Taylor, 2016: Numerical simulations of the equilibrium between eddy-induced restratification and vertical mixing. J. Phys. Oceanogr., 46, 919-935, https://doi.org/ 10.1175/JPO-D-15-0110.1.

Boccaletti, G., R. Ferrari, and B. Fox-Kemper, 2007: Mixed layer instabilities and restratification. J. Phys. Oceanogr., 37, 2228-2250, https://doi.org/10.1175/JPO3101.1.

Bretherton, F. P., and M. Karweit, 1975: Mid-ocean mesoscale modeling. Numerical Models of Ocean Circulation, R. O. Reid, A. R. Robinson, and K. Bryan, Eds., National Academy of Sciences, 237-249.

Callies, J., and R. Ferrari, 2018: Baroclinic instability in the presence of convection. J. Phys. Oceanogr., 48, 45-60, https:// doi.org/10.1175/JPO-D-17-0028.1.

- G. Flierl, R. Ferrari, and B. Fox-Kemper, 2016: The role of mixed-layer instabilities in submesoscale turbulence. J. Fluid Mech., 788, 5-41, https://doi.org/10.1017/jfm.2015.700.

Capet, X., E. J. Campos, and A. M. Paiva, 2008: Submesoscale activity over the Argentinian shelf. Geophys. Res. Lett., 35, L15605, https://doi.org/10.1029/2008GL034736.

Emanuel, K. A., 1994: Atmospheric Convection. Oxford University Press, 580 pp.

Fox-Kemper, B., and R. Ferrari, 2008: Parameterization of mixed layer eddies. Part II: Prognosis and impact. J. Phys. Oceanogr., 38, 1166-1179, https://doi.org/10.1175/2007JPO3788.1.

,-- , and R. W. Hallberg, 2008: Parameterization of mixed layer eddies. Part I: Theory and diagnosis. J. Phys. Oceanogr., 38, 1145-1165, https://doi.org/10.1175/2007JPO3792.1. 
and Coauthors, 2011: Parameterization of mixed layer eddies. III: Implementation and impact in global ocean climate simulations. Ocean Modell., 39, 61-78, https://doi.org/10.1016/ j.ocemod.2010.09.002.

Garner, S. T., N. Nakamura, and I. M. Held, 1992: Nonlinear equilibration of two-dimensional Eady waves: A new perspective. J. Atmos. Sci., 49, 1984-1996, https://doi.org/10.1175/ 1520-0469(1992)049<1984:NEOTDE > 2.0.CO;2.

Gent, P. R., and J. C. McWilliams, 1990: Isopycnal mixing in ocean circulation models. J. Phys. Oceanogr., 20, 150-155, https:// doi.org/10.1175/1520-0485(1990)020<0150:IMIOCM > 2.0.CO;2.

_ , and Coauthors, 2011: The Community Climate System Model version 4. J. Climate, 24, 4973-4991, https://doi.org/ 10.1175/2011JCLI4083.1.

Haidvogel, D. B., and I. M. Held, 1980: Homogeneous quasigeostrophic turbulence driven by a uniform temperature gradient. J. Atmos. Sci., 37, 2644-2660, https://doi.org/10.1175/ 1520-0469(1980)037<2644:HQGTDB>2.0.CO;2.

Haine, T. W. N., and J. Marshall, 1998: Gravitational, symmetric, and baroclinic instability of the ocean mixed layer. J. Phys. Oceanogr., 28, 634-658, https://doi.org/10.1175/1520-0485 (1998)028<0634:GSABIO > 2.0.CO;2.

Juckes, M. N., 1998: Baroclinic instability of semi-geostrophic fronts with uniform potential vorticity. I: An analytic solution. Quart. J. Roy. Meteor. Soc., 124, 2227-2257, https:// doi.org/10.1002/qj.49712455104.

Larichev, V. D., and I. M. Held, 1995: Eddy amplitudes and fluxes in a homogeneous model of fully developed baroclinic instability. J. Phys. Oceanogr., 25, 2285-2297, https://doi.org/ 10.1175/1520-0485(1995)025<2285:EAAFIA $>2.0 . C O ; 2$.

Mahadevan, A., E. D'Asaro, C. Lee, and M. J. Perry, 2012: Eddy-driven stratification initiates North Atlantic spring phytoplankton blooms. Science, 337, 54-58, https://doi.org/ 10.1126/science. 1218740 .

Manucharyan, G. E., and M.-L. Timmermans, 2013: Generation and separation of mesoscale eddies from surface ocean fronts. J. Phys. Oceanogr., 43, 2545-2562, https://doi.org/10.1175/ JPO-D-13-094.1.

Marshall, J., A. Adcroft, C. Hill, L. Perelman, and C. Heisey, 1997: A finite-volume, incompressible Navier Stokes model for studies of the ocean on parallel computers. J. Geophys. Res., 102, 5753-5766, https://doi.org/10.1029/96JC02775.

Salmon, R., 1980: Baroclinic instability and geostrophic turbulence. Geophys. Astrophys. Fluid Dyn., 15, 167-211, https:// doi.org/10.1080/03091928008241178.

Sasaki, H., P. Klein, B. Qiu, and Y. Sasai, 2014: Impact of oceanicscale interactions on the seasonal modulation of ocean dynamics by the atmosphere. Nat. Commun., 5, 5636, https:// doi.org/10.1038/ncomms6636.

Stone, P. H., 1966: On non-geostrophic baroclinic stability. J. Atmos. Sci., 23, 390-400, https://doi.org/10.1175/1520-0469(1966)023<0390: ONGBS $>2.0 . \mathrm{CO} ; 2$

1970: On non-geostrophic baroclinic stability: Part II. J. Atmos. Sci. 27, 721-726, https://doi.org/10.1175/1520-0469(1970)027<0721: ONGBSP $>2.0 . \mathrm{CO} ; 2$.

__ 1971: Baroclinic stability under non-hydrostatic conditions. J. Fluid Mech., 45, 659-671, https://doi.org/10.1017/ S0022112071000260.

Taylor, J. R., and R. Ferrari, 2009: On the equilibration of a symmetrically unstable front via a secondary shear instability. J. Fluid Mech., 622, 103, https://doi.org/10.1017/S0022112008005272.

, and 2010: Buoyancy and wind-driven convection at mixed layer density fronts. J. Phys. Oceanogr., 40, 1222-1242, https://doi.org/10.1175/2010JPO4365.1. 